\title{
Optimal Error Properties of Finite Element Methods for Second Order Elliptic Dirichlet Problems
}

\author{
By Arthur G. Werschulz
}

\begin{abstract}
We use the informational approach of Traub and Woźniakowski [9] to study the variational form of the second order elliptic Dirichlet problem $L u=f$ on $\Omega \subset \mathbf{R}^{N}$. For $f \in H^{r}(\Omega)$, where $r \geqslant-1$, a quasi-uniform finite element method using $n$ linear functionals $\int_{\Omega} f \psi_{i}$ has $H^{1}(\Omega)$-norm error $\Theta\left(n^{-(r+1) / N}\right)$. We prove that it is asymptotically optimal among all methods using any information consisting of any $n$ linear functionals. An analogous result holds if $L$ is of order $2 m$ : if $f \in H^{r}(\Omega)$, where $r \geqslant-m$, then there is a finite element method whose $H^{\alpha}(\Omega)$-norm error is $\Theta\left(n^{-(2 m+r-\alpha) / N}\right)$ for $0 \leqslant \alpha \leqslant m$, and this is asymptotically optimal; thus, the optimal error improves as $m$ increases. If the integrals $\int_{\Omega} f \psi_{i}$ are approximated by using $n$ evaluations of $f$, then there is a finite element method with quadrature with $H^{1}(\Omega)$-norm error $O\left(n^{-r / N}\right)$ where $r>N / 2$. We show that when $N=1$, there is no method using $n$ function evaluations whose error is better than $\Omega\left(n^{-r}\right)$; thus for $N=1$, the finite element method with quadrature is asymptotically optimal among all methods using $n$ evaluations of $f$.
\end{abstract}

1. Introduction. This paper deals with the optimal solution of second order elliptic partial differential equations. We wish to consider the variational form of the problem

$$
L u=f \quad \text { in } \Omega \subset \mathbf{R}^{N}, \quad u=0 \text { on } \partial \Omega,
$$

(see Section 2). Suppose that we evaluate information of the form

$$
\left\{\int_{\Omega} f \psi_{1}, \ldots, \int_{\Omega} f \psi_{n}\right\} .
$$

If $f \in H^{r}(\Omega)$, where $r \geqslant-1$, there exists a finite element method using (1.2) whose error is $O\left(n^{-(r+1) / N}\right)$ when measured in the $H^{1}(\Omega)$ norm.

We first wish to answer two questions. First, is there another method using the information (1.2) whose error is better than that of this finite element method? Second, is the information (1.2) the best possible information using $n$ linear functionals? That is, is there another set of $n$ linear functionals such that the best algorithm using this new information is better than the best algorithm using (1.2)? In Section 3, we asymptotically answer these questions in the negative. Thus, this finite element method is of asymptotically optimal error among all algorithms, linear or nonlinear, using any $n$ linear functionals whatsoever.

We also report some results on $2 m$ th order elliptic problems which indicate that as $m$ increases, the same number of evaluations yields smaller $H^{m}(\Omega)$ error.

Received May 20, 1980; revised June 15, 1981.

1980 Mathematics Subject Classification. Primary 65N30, 68C25; Secondary 65J10, 65N05, 65N15. 
Although this is true, the cost of constructing an approximation of given accuracy increases with $m$. In addition, we consider the case where the error is measured by other Sobolev norms.

In many cases, the information (1.2) is not available. Then $\int_{\Omega} f \psi_{i}$ is approximated via a quadrature rule using function evaluations. Thus, instead of using the "optimal" information of size $n$, we use the standard information

$$
\left\{f\left(x_{1}\right), \ldots, f\left(x_{n}\right)\right\}
$$

of size $n$. If $f \in H^{r}(\Omega), r>N / 2$, there exists a finite element method with quadrature using (1.2) with $x_{i}=x_{i}^{* *}$ whose error is $O\left(n^{-r / N}\right)$.

We now pose two additional questions. First, is there another method using $\left\{f\left(x_{1}^{* *}\right), \ldots, f\left(x_{n}^{* *}\right)\right\}$ whose error is better than this finite element method with quadrature? Second, is there a choice of $x_{i}$ in (1.3) which yields better error than the choice $x_{i}=x_{i}^{* *}$ ? In Section 4, we asymptotically answer these questions in the negative for the case $N=1$. That is, the finite element method with quadrature is asymptotically of optimal error among all algorithms using information of the form (1.3), and the choice $x_{i}=x_{i}^{* *}$ is asymptotically optimal. Since we have this negative result for a particular value of $N$, there is no way of improving the error behavior for all $N$.

In what follows, we use the $\Omega$ and $\Theta$ notations, as well as the more commonly used $O$ and $o$ notations. We say that $f(n)=\Omega(g(n))$ if $g(n)=O(f(n))$, and $f(n)=$ $\Theta(g(n))$ if $f(n)=O(g(n))$ and $f(n)=\Omega(g(n))$.

2. The Variational Dirichlet Problem. In what follows, we use the standard notation for Sobolev spaces, norms, etc., found in Ciarlet [3]. Let $\Omega$ be a $C^{\infty}$ region in $\mathbf{R}^{N}$, and let $A: \Omega \rightarrow \mathbf{R}^{N \times N}$ and $q: \Omega \rightarrow \mathbf{R}^{N}$ be given $C^{\infty}$ mappings. We assume that $A$ is uniformly positive definite, i.e., $A$ is symmetric and there exists $\gamma>0$ such that

$$
\xi^{T} A(x) \xi \geqslant \gamma \xi^{T} \xi, \quad \forall \xi \in \mathbf{R}^{N}, \forall x \in \Omega .
$$

We also assume that $q(x) \geqslant 0$ for $x \in \Omega$.

We define a bilinear form $B: H_{0}^{1}(\Omega) \times H_{0}^{1}(\Omega) \rightarrow \mathbf{R}$ by

$$
B(v, w):=\int_{\Omega}\left[(A \nabla v)^{T} \nabla w+q v w\right] .
$$

Recall that $H^{-1}(\Omega)$ is the completion of $C^{\infty}(\Omega)$ under the norm

$$
\|g\|_{-1}:=\sup _{\substack{\psi \in H^{1}(\Omega) \\ \psi \neq 0}} \frac{\int_{\Omega} g \psi}{\|\psi\|_{1}},
$$

see pp. 18 ff. of Babuška and Aziz [2].

Then the variational Dirichlet problem is defined as follows. Let $r \geqslant-1$. For a given $f \in H^{r}(\Omega)$, find $u=S f \in H_{0}^{1}(\Omega)$ satisfying

$$
B(u, v)=(f, v):=\int_{\Omega} f v \quad \forall v \in H_{0}^{1}(\Omega) .
$$

Using the assumptions on $A$ and $q$, the Lax-Milgram theorem implies that $S$ : $H^{r}(\Omega) \rightarrow H_{0}^{1}(\Omega)$ is a bounded linear operator, and is a Hilbert space isomorphism of $H^{-1}(\Omega)$ onto $H_{0}^{1}(\Omega)$. 
For future reference, we recall the "shift theorem" (see Chapter 3 of Babuška and Aziz [2]) which states that since $f \in H^{r}(\Omega), S f \in H_{0}^{1}(\Omega) \cap H^{r+2}(\Omega)$; moreover, there is a positive constant $\sigma$, independent of $f$, for which

$$
\sigma^{-1}\|S f\|_{r+2} \leqslant\|f\|_{r} \leqslant \sigma\|S f\|_{r+2} .
$$

If $r>N / 2$, then the shift theorem and the Sobolev embedding theorem yield that the solution $u=S f$ to the variational Dirichlet problem will be the solution to the classical Dirichlet problem, that is,

$$
-\operatorname{div}(A \nabla u)+q u=f \text { in } \Omega
$$

and

$$
u=0 \quad \text { on } \partial \Omega \text {. }
$$

3. Asymptotically Optimal Error Algorithms Using General Information. We first consider a class of finite element methods for the problem (2.4). For further details, see Babuška and Aziz [2] or Ciarlet [3]. These methods have the property that their explicit dependence on $f$ in (2.4) is actually through the information

$$
\Re_{n}^{*} f:=\left[\left(f, \psi_{1}^{n}\right) \cdots\left(f, \psi_{n}^{n}\right)\right]^{T},
$$

where the functions $\psi_{i}^{n}: \bar{\Omega} \rightarrow \mathbf{R}$ will be defined below.

Given $r$ as in Section 2, we define the integer $k$ by

$$
k:=\lceil r\rceil+1 \text {, }
$$

and let $\left\{\mathcal{S}_{h}\right\}_{h>0}$ be a quasi-uniform family of finite element subspaces of $H_{0}^{1}(\Omega)$ of degree $k$. That is, there is a quasi-uniform family $\left\{\sigma_{h}\right\}_{h>0}$ of triangulations of $\bar{\Omega}$ such that $v_{h} \in \mathcal{S}_{h}$ if and only if $v_{h}$ is continuous, $v_{h}$ vanishes on $\partial \Omega$, and $\left.v_{h}\right|_{E} \in \mathscr{P}_{k}$ for each subregion $E$ of $\mathcal{T}_{h}$. Here,

$$
\mathscr{P}_{k}:=\operatorname{span}\left\{x_{1}^{\alpha_{1}} \cdots x_{N}^{\alpha_{N}}: \alpha_{i} \geqslant 0 \text { integer, } \sum_{i=1}^{N} \alpha_{i} \leqslant k\right\} \text {. }
$$

(Of course, since we have assumed that $\Omega$ is $C^{\infty}$, we must make an additional assumption about the boundary elements to guarantee that $\varsigma_{h} \subset H_{0}^{1}(\Omega)$; for example, we may use curved elements as in [4].) Let

$$
n=n(h):=\operatorname{dim} \varsigma_{h} .
$$

We choose the degrees of freedom of the finite element space to consist of function values at a set $p_{1}^{n}, \ldots, p_{n}^{n} \in \bar{\Omega}$ of nodes. Then any $v_{h} \in \mathcal{S}_{h}$ may be written uniquely as

$$
v_{h}(x)=\sum_{j=1}^{n} v_{h}\left(p_{j}^{n}\right) \psi_{j}^{n}(x)
$$

where the basis functions $\psi_{1}^{n}, \ldots, \psi_{n}^{n}$ are chosen so that $\psi_{j}^{n}\left(p_{i}^{n}\right)=\delta_{i j}$, so that they have "small" support. The quasi-uniformity implies that there exists $C>0$ for which

$$
h \leqslant C n^{-1 / N} \text {. }
$$

We briefly recall the approximation properties of the finite element space. Let $v \in H^{r+2}(\Omega)$. We then define the $\delta_{h}$-projection $P_{h} v$ of $v$ by

$$
\left\|v-P_{h} v\right\|_{1}=\inf _{v_{h} \in S_{h}}\left\|v-v_{h}\right\|_{1} \text {. }
$$


Then there is a $C>0$, independent of $v$ and $h$, such that

$$
\left\|v-P_{h} v\right\|_{1} \leqslant C h^{r+1}\|v\|_{r+2} \text {. }
$$

The finite element method $\varphi_{n}^{*}$ is defined as follows. Given $f \in H^{r}(\Omega)$, define $u_{h}^{*} \in \mathcal{S}_{h}$ for $h>0$ by

$$
B\left(u_{h}^{*}, v_{h}\right)=\left(f, v_{h}\right) \quad \forall v_{h} \in \mathcal{S}_{h} .
$$

Writing

$$
u_{h}^{*}(x)=\sum_{j=1}^{n} u_{j} \psi_{j}^{n}(x),
$$

we see that $\mathbf{u}=\left[u_{1} \cdots u_{n}\right]^{T}$ is the solution to

$$
\mathbf{K u}=\mathbf{b},
$$

where the entries $K_{i j}$ and $b_{i}$ of the matrix $\mathbf{K}$ and the vector $\mathbf{b}$ are, respectively, given by

$$
K_{i j}=B\left(\psi_{j}^{n}, \psi_{i}^{n}\right), \quad b_{i}=\left(f, \psi_{i}\right) .
$$

Since the dependence of $u_{h}^{*}$ on $f$ is only through the information (3.1), the finite element method

$$
\varphi_{n}^{*}\left(\mathcal{T}_{n}^{*} f\right):=u_{h}^{*}
$$

where $n=\operatorname{dim} \delta_{n}$, is well defined.

We now consider the error of the finite element method. Since (2.4) and (3.9) yield

$$
\left\|u-u_{h}^{*}\right\|_{1} \leqslant C \inf _{v_{h} \in \delta_{h}}\left\|u-v_{h}\right\|_{1}=C\left\|u-P_{h} u\right\|_{1}
$$

(for positive $C$ independent of $u$ and $h$ ), we may use (2.5) and (3.8) to find that there is a $C>0$ for which

$$
\left\|u-u_{h}^{*}\right\|_{1} \leqslant C h^{r+1}\|f\|_{r} .
$$

So (3.6) yields a positive $\beta$, independent of $n$ and $f$, for which

$$
\left\|S f-\varphi_{n}^{*}\left(\mathcal{X}_{n}^{*} f\right)\right\|_{1}=\left\|u-u_{h}^{*}\right\|_{1} \leqslant \beta n^{-(r+1) / N}\|f\|_{r} .
$$

We are now ready to consider the two main problems of this section.

(i) Is there any other "algorithm" (method) using the information (3.1) whose error behavior is better than that of the finite element method?

(ii) Is there another choice of "information" using $n$ linear functionals of $f$ which is better than the information (3.1)? That is, is there an algorithm using this other information and having better error than the best algorithm using (3.1)?

In order to answer these questions, we must specify our intuitive ideas of "algorithm" and "information" more precisely. We use the notions and results found in Part A of Traub and Woźniakowski [9], hereafter referred to as "Part A."

By information of cardinality $n$, we mean a linear operator

$$
\Re: H^{r}(\Omega) \rightarrow \mathbf{R}^{n}
$$

such that

$$
\Re f=\left[L_{1} f \cdots L_{n} f\right]^{T}
$$


for some linear functionals $L_{1}, \ldots, L_{n}$ on $H^{r}(\Omega)$. Clearly the information $\mathscr{T}_{n}^{*}$ given by (3.1) is of cardinality $n$.

Let $\mathscr{F}_{0}$ be the unit ball of $H^{r}(\Omega)$, i.e.,

$$
\mathscr{F}_{0}=B H^{r}(\Omega):=\left\{f \in H^{r}(\Omega):\|f\|_{r} \leqslant 1\right\} .
$$

An algorithm $\varphi$ using the information $\Re$ is a (not-necessarily linear) operator $\varphi$ : $\Re\left(\mathscr{F}_{0}\right) \rightarrow H_{0}^{1}(\Omega)$. This means that $\varphi$ approximates $S f$ for $f \in \mathscr{F}_{0}$ by using only the information $\Re f$.

The (worst-case) error of an algorithm $\varphi$ using $\Re$ is defined to be

$$
e(\varphi):=\sup _{f \in \mathscr{F}_{0}}\|S f-\varphi(\Re f)\|_{1} .
$$

Results from Chapters 2 and 3 of Part A yield

$$
\inf _{\varphi} e(\varphi)=r\left(\mathscr{T}, S, \mathscr{F}_{0}\right):=\sup _{z \in \operatorname{ker} \mathscr{T} \cap \mathscr{F}_{0}}\|S z\|_{1},
$$

where the infimum is over all $\varphi$ using $\Re$ and $r\left(\mathcal{K}, S, \mathscr{F}_{0}\right)$ is called the radius of information. We say that $\varphi$ is an optimal error algorithm using $\Re$ if $\varphi$ uses $\Re$ and

$$
e(\varphi)=r\left(\Re, S, \mathscr{F}_{0}\right)
$$

Our second task is to determine, for each positive integer $n$, the most "relevant" information of cardinality $n$. Define the $n$th minimal radius of information by

$$
r\left(n, S, \mathscr{F}_{0}\right):=\inf _{\Re} r\left(\Re, S, \mathscr{F}_{0}\right),
$$

where the infimum is over all $\mathscr{\Re}$ of cardinality $n$. Then $\mathscr{\Re}$ of cardinality $n$ is an $n$th optimal information if

$$
r\left(\Re, S, \mathscr{F}_{0}\right)=r\left(n, S, \mathscr{F}_{0}\right) .
$$

Recall that for a balanced subset $X$ of a Hilbert space $H$, the Kolmogorov $n$-width $d_{n}(X, H)$ is defined to be

$$
d_{n}(X, H):=\inf _{A_{n}} \sup _{x \in X} \inf _{y \in A_{n}}\|x-y\|_{H},
$$

where $A_{n}$ is a subspace of $H$ with dimension at most $n$. Using results from Chapters 2 and 3 of Part $A$, it is easy to see that

$$
r\left(n, S, \mathscr{F}_{0}\right)=d_{n}\left(S\left(\mathscr{F}_{0}\right), H_{0}^{1}(\Omega)\right)
$$

(This may also be viewed as a consequence of the equality of the Kolmogorov and Gelfand $n$-widths in a reflexive Banach space.)

We are now ready to prove the main result of this section.

THEOREM 3.1. Let $\mathscr{F}_{0}$ be the unit ball of $H^{r}(\Omega), r \geqslant-1$. Then the following hold:

(i) $r\left(n, S, \mathscr{F}_{0}\right)=\Theta\left(n^{-(r+1) / N}\right)$ as $n \rightarrow \infty$.

(ii) The information $\mathfrak{T}_{n}^{*}$ given by (3.1) is an nth asymptotically optimal information (i.e., optimal to within a constant factor), and

$$
r\left(\Re_{n}^{*}, S, \mathscr{F}_{0}\right)=\Theta\left(n^{-(r+1) / N}\right) \text { as } n \rightarrow \infty .
$$

(iii) The finite element method $\varphi_{n}^{*}$ given by (3.13) is an asymptotically optimal algorithm using $\mathfrak{X}_{n}^{*}$ and

$$
e\left(\varphi_{n}^{*}\right)=\Theta\left(n^{-(r+1) / N}\right) .
$$


Proof. Let

$$
X:=\left\{u \in H_{0}^{r+2}(\Omega):\|u\|_{r+2} \leqslant \sigma^{-1}\right\},
$$

where $\sigma$ is from (2.5). We claim that

$$
X \subset S\left(\mathscr{F}_{0}\right) .
$$

Indeed, let $u \in X$. Since $r \geqslant-1, u \in H_{0}^{1}(\Omega) \cap H^{r+2}(\Omega)$, and there exists a unique $f \in H^{r}(\Omega)$ such that $S f=u$. Moreover,

$$
\|u\|_{r+2} \leqslant \sigma^{-1}
$$

so that the shift theorem (2.5) yields

$$
\|f\|_{r} \leqslant \sigma\|S f\|_{r+2} \leqslant 1
$$

i.e., $f \in \mathscr{F}_{0}$. Thus $u=S f \in S\left(\mathscr{F}_{0}\right)$, i.e., (3.28) holds as claimed. Since for any Hilbert space $H$ and any balanced subsets $X$ and $Y$ of $H$ we have

$$
X \subseteq Y \Rightarrow d_{n}(X, H) \leqslant d_{n}(Y, H),
$$

we see that (3.28) implies

$$
d_{n}\left(S\left(\mathscr{F}_{0}\right), H_{0}^{\mathrm{l}}(\Omega)\right) \geqslant d_{n}\left(X, H_{0}^{\mathrm{l}}(\Omega)\right)=\sigma^{-1} d_{n}\left(B H_{0}^{r+2}(\Omega), H_{0}^{1}(\Omega)\right) .
$$

Using a proof analogous to that of Theorem 2.5.1 of Babuska and Aziz [2], there is a positive constant $C$, independent of $n$, such that

$$
d_{n}\left(B H_{0}^{r+2}(\Omega), H_{0}^{1}(\Omega)\right) \geqslant C\left[d_{n}\left(B H_{0}^{1}(\Omega), L_{2}(\Omega)\right)\right]^{r+1} .
$$

Jerome [5], [6] uses a result on pp. 250-251 of Agmon [1] to show

$$
d_{n}\left(B H_{0}^{1}(\Omega), L_{2}(\Omega)\right) \geqslant\left(C^{\prime} n\right)^{-1 / N}(1+o(1)),
$$

where

$$
C^{\prime}=\frac{\operatorname{vol}(\Omega)}{2^{N} \pi^{N / 2} \Gamma(1+N / 2)},
$$

$\Gamma$ denoting the gamma function. Thus (3.26), (3.32), (3.33), and (3.34) imply that there exists a positive constant $\alpha$, independent of $n$, such that

$$
r\left(n, S, \mathscr{F}_{0}\right) \geqslant \alpha n^{-(r+1) / N} \text {. }
$$

On the other hand, (3.13), (3.16), (3.19), and (3.20) imply that

$$
e\left(\varphi_{n}^{*}\right) \leqslant \beta n^{-(r+1) / N} .
$$

The theorem follows from (3.36) and (3.37).

Thus the finite element method using $n$ evaluations of $\left(f, \psi_{i}^{n}\right)$ is (asymptotically) of optimal error among all algorithms using any information of cardinality $n$.

Remark 3.1. Consider the case $r=-1$. Then $\mathscr{F}_{0}$ is the unit ball of $H^{-1}(\Omega)$. Note that $H^{-1}(\Omega)$ is the largest space for which a solution in $H_{0}^{1}(\Omega)$ is guaranteed. However, there exists $C>0$, independent of $n$, such that

$$
r\left(n, S, B H^{-1}(\Omega)\right) \geqslant C .
$$

Thus in the case where the problem data have only enough smoothness to guarantee that the solution operator $S$ is well defined and bounded. the problem is not 
convergent, in the sense that for no sequence $\left\{\varphi_{n}\right\}_{n=1}^{\infty}$ of algorithms, with $\varphi_{n}$ using any information of cardinality $n$, do we have

$$
\lim _{n \rightarrow \infty} e\left(\varphi_{n}\right)=0 \text {. }
$$

More generally, it may be shown that if $\mathscr{F}_{0}$ is the unit ball of a Hilbert space $H$ which is embedded in $H^{-1}(\Omega)$, then the problem is convergent (in the sense that (3.39) holds for some sequence of algorithms) if and only if the embedding of $H$ into $H^{-1}(\Omega)$ is compact.

Remark 3.2. The information operator $\Re$ given by (3.9) is defined by $n$ linear functionals which are given independent of the choice of problem datum $f$. It is possible to consider "adaptive" information operators for which the evaluation of the $i$ th functional depends on the previous $i-1$ evaluations. However, there is no advantage in doing this; when $\mathscr{F}_{0}$ is the unit ball of a Hilbert space, the $n$th minimal radius is the same for nonadaptive information of the form (3.9) and for adaptive information. (See Section 2.7 of Part A.)

Remark 3.3. We generalize this problem by considering a $2 m$ th order elliptic problem and measuring the error in the $H^{\alpha}(\Omega)$ norm $(0 \leqslant \alpha \leqslant m)$. Then for $r \geqslant-m$, a finite element algorithm using subspaces of $H_{0}^{m}(\Omega)$ which are piecewise polynomials of degree $[r]+2 m-1$ is asymptotically optimal, and

$$
r\left(n, S, B H^{r}(\Omega)\right)=\Theta\left(n^{-(2 m+r-\alpha) / N}\right) \quad \text { as } n \rightarrow \infty .
$$

This holds because the shift theorem for a $2 m$ th order elliptic operator takes the form

$$
\sigma^{-1}\|f\|_{r} \leqslant\|S f\|_{r+2 m} \leqslant \sigma\|f\|_{r} \quad \forall f \in H^{r}(\Omega) .
$$

Hence the asymptotic error improves whenever $\alpha$ decreases, $m$ increases, $N$ decreases, or $r$ increases.

Remark 3.4. For further information on optimal interpolation-error estimates and elliptic problems, the reader may wish to consult Schultz [7].

We now consider the computational complexity of using the finite element method $\varphi_{n}^{*}$ to find an $\varepsilon$-approximation to $S$, that is, of computing the value of $\varphi_{n}^{*}\left(\mathcal{T}_{n}^{*} f\right)$ such that

$$
e\left(\varphi_{n}^{*}\right) \leqslant \varepsilon .
$$

By (iii) of Theorem 3.1, we must choose

$$
n=n^{*}(\varepsilon)=\Theta\left(\varepsilon^{-N /(r+1)}\right)
$$

in order to guarantee (3.42). The algorithm $\varphi_{n}^{*}$ is linear, i.e., of the form

$$
\varphi_{n}^{*}\left(\Re_{n}^{*} f\right)=\sum_{i=1}^{n}\left(f, \psi_{i}^{n}\right) g_{i},
$$

where $g_{i} \in H_{0}^{1}(\Omega)$ are independent of $f$. If one agrees to use preconditioning, i.e., to compute the $g_{i}$ in advance and not count the cost of this precomputation, then the computation of $\varphi_{n}^{*}\left(\Re_{n}^{*} f\right)(x)$ at any point $x \in \Omega$ requires the computation of $n$ inner products and $2 n-1$ arithmetic operations. Assuming that the inner products can be computed in finite time, we conclude that the complexity of using the finite element method to compute an $\varepsilon$-approximation is

$$
\operatorname{COMP}\left(\varphi_{n}^{*}, \varepsilon\right)=\Theta\left(\varepsilon^{-N /(r+1)}\right) \quad \text { as } \varepsilon \rightarrow 0,
$$


where $n$ is given by (3.43). Since $\varphi_{n}^{*}$ is (to within a constant factor) an optimal error algorithm, it follows that

$$
\operatorname{COMP}(S, \varepsilon)=\inf _{\varphi} \operatorname{comp}(\varphi, \varepsilon)=\Theta\left(\varepsilon^{-N /(r+1)}\right) .
$$

Thus the finite element method is (to within a constant factor) of optimal complexity.

We wish to comment on the preconditioning assumption. In practice, such preconditioning is not often done. One reason is that for large $n$, the complexity of the precomputation may be prohibitively expensive, since it essentially amounts to computing the $L U$-factorization of the matrix $\mathbf{K}$ of (3.11), while not counting the cost of this factorization. Another reason is that one often wishes to fix $f$ and let $n$ increase (e.g., in Richardson extrapolation); in this case, one cannot take advantage of the linearity of $\varphi_{n}^{*}$.

The computation of $\varphi_{n}^{*}$ now requires the solution of the large sparse linear system (3.11). This is, in itself, an active area of research. However, for $N=1$, the stiffness matrix $\mathbf{K}$ in (3.11) is a banded matrix whose bandwidth depends only on $r$, and not on $n$; this system can be solved in $\Theta(n)$ operations, even if precomputing is not allowed. So for the case $N=1$, the result (3.45) still holds, and the finite element method is still an asymptotically optimal-complexity algorithm.

Remark 3.5. Similarly, one can show that if preconditioning is allowed, there is a finite element method for a $2 m$ th order elliptic problem which computes an $\varepsilon$-approximation in the $H^{\alpha}(\Omega)$ norm $(0 \leqslant \alpha \leqslant m)$ with complexity $\Theta\left(\varepsilon^{-N /(2 m+r-\alpha)}\right)$, and that this is an asymptotically optimal-complexity algorithm for computing an $\varepsilon$-approximation. Hence, the complexity decreases as $m$ increases and $\alpha$ decreases. However, if one does not want to use preconditioning, this optimal complexity is no longer apparent, since the combinatory complexity increases with $m$.

4. Asymptotically Optimal Error Algorithms Using Standard Information. The asymptotically optimal algorithms $\varphi_{n}^{*}$ described in the last section require the calculation of the inner products

$$
\left(f, \psi_{j}^{n}\right)=\int_{\Omega} f \psi_{j}^{n} \quad(1 \leqslant j \leqslant n)
$$

for $f \in H^{r}(\Omega)$. In practice, this is often accomplished by replacing the integral with a quadrature rule using function evaluations $f\left(x_{i}\right), x_{i} \in \bar{\Omega}$. Recall that the Sobolev embedding theorem requires that $r>N / 2$ in order for $f\left(x_{i}\right)$ to be defined for all $f \in H^{r}(\Omega)$.

In this section, we use $r$ from Section 2 to define an integer $k$ by

$$
k:=\lceil r\rceil \text {. }
$$

Chapter 4.1 of [3] considers the use of a quadrature rule which is exact for piecewise polynomials of degree at most $2 k-2$ to approximate the inner products occurring in a finite element method using a quasi-uniform family $\left\{\delta_{h}\right\}_{h>0}$. Here $\delta_{h}$ is a finite element subspace of $H_{0}^{1}(\Omega)$ of degree $k$; the degrees of freedom of $\delta_{h}$ once again consist of function evaluations at nodes in $\bar{\Omega}$. Denoting the approximate solution thus produced by $u_{h}^{* *}$, Ciarlet [3] shows that there is a positive constant $C$, independent of $u$ and $h$, such that

$$
\left\|u-u_{h}^{* *}\right\|_{1} \leqslant C h^{r}\|f\|_{r} .
$$


The dependence of $u_{h}^{* *}$ on $f$ is only through the information

$$
\Re_{n}^{* *} f:=\left[f\left(x_{1}^{* *}\right) \cdots f\left(x_{n}^{* *}\right)\right]^{T}
$$

where

$$
n=\Theta\left(\operatorname{dim} \delta_{h}\right)
$$

The finite element method with quadrature

$$
\varphi_{n}^{* *}\left(\Re_{n}^{* *} f\right):=u_{h}^{* *}
$$

is well defined. Moreover, if we let $\mathscr{F}_{0}=B H^{r}(\Omega)$ be the unit ball of $H^{r}(\Omega)$ (see (3.19)), then (3.20), (4.3), (4.6), and the quasi-uniformity of $\left\{\delta_{h}\right\}_{h>0}$ imply that there exists a positive constant $\beta$, independent of $n$, such that

$$
e\left(\varphi_{n}^{* *}\right) \leqslant \beta n^{-r / N} \text {. }
$$

Note that this error bound is worse than the optimal error when using the information $\Re_{n}^{*}$. This leads us to consider the main problems of this section.

(i) Is there any other algorithm using $\Re_{n}^{* *}$ whose error is better than that of $\varphi_{n}^{* *}$ ?

(ii) Is there another choice of the points $x_{i}^{* *}$ in (4.4) which yields an optimal error algorithm with smaller error?

We show that (i) and (ii) cannot be generally answered in the affirmative, by considering the case $N=1$. In this case, $\Omega$ becomes an open interval $I$ on the real line. Defining, for $x_{1}, \ldots, x_{n} \in \bar{I}$, the standard information $\mathscr{T}_{n}$ by

$$
\Re_{n} f:=\left[f\left(x_{1}\right) \cdots f\left(x_{n}\right)\right]^{T},
$$

we have

THEOREM 4.1. Let I be a real interval, and let $\mathscr{F}_{0}$ be the unit ball of $H^{r}(I), r>\frac{1}{2}$. Then the following hold:

(i) For any $x_{1}, \ldots, x_{n} \in \bar{I}$,

$$
r\left(\Re_{n}, S, \mathscr{F}_{0}\right)=\Omega\left(n^{-r}\right) \text { as } n \rightarrow \infty .
$$

(ii) The points $x_{i}=x_{i}^{* *}$ are asymptotically optimal, i.e.,

$$
r\left(\mathscr{T}_{n}^{* *}, S, \mathscr{F}_{0}\right)=\Theta\left(\inf _{x_{1}, \ldots, x_{n} \in \bar{I}} r\left(\mathcal{X}_{n}, S, \mathscr{F}_{0}\right)\right)=\Theta\left(n^{-r}\right) \text { as } n \rightarrow \infty \text {. }
$$

(iii) The finite element method with quadrature, $\varphi_{n}^{* *}$, is an asymptotically optimal error using $\Re_{n}^{* *}$, and

$$
e\left(\varphi_{n}^{* *}\right)=\Theta\left(n^{-r}\right) \text { as } n \rightarrow \infty
$$

Proof. Since $S$ is a Hilbert space isomorphism of $H^{-1}(I)$ onto $H_{0}^{1}(\Omega)$, and $H^{r}(I) \subseteq H^{-1}(I)$, there is a positive constant $C$ such that

$$
\|S z\|_{1} \geqslant C\|z\|_{-1} \quad \forall z \in H^{r}(I) .
$$

Using (2.3) and choosing $\psi \in H^{1}(I)$ to be the constant function $\psi(x) \equiv 1$, we see that

$$
\|z\|_{-1} \geqslant|I|^{-1 / 2}\left|\int_{I} z\right| \quad \forall z \in H^{r}(\Omega),
$$


$|I|$ denoting the length of $I$. Let $x_{1}, \ldots, x_{n} \in \bar{I}$ be given. Then (3.21), (4.9), and (4.10) imply that there is a positive constant $C$, independent of $n$ and the selection of $x_{1}, \ldots, x_{n} \in \bar{I}$, such that

$$
r\left(\mathscr{\Re}_{n}, S, \mathscr{F}_{0}\right) \geqslant C\left|\int_{\Omega} z\right|
$$

for any $z \in \operatorname{ker} \mathscr{T}_{n} \cap \mathscr{F}_{0}$.

We now define a function $z \in \operatorname{ker} \mathscr{X}_{n} \cap \mathscr{F}_{0}$ which gives the desired result. Let $x_{0}$ and $x_{n+1}$ denote the left and right endpoints of $I$, and assume without loss of generality that $x_{1}<x_{2}<\cdots<x_{n}$. Define

$$
h_{i}:=x_{i+1}-x_{i} \quad(0 \leqslant i \leqslant n)
$$

and

$$
p_{i}:=\frac{1}{2}\left(x_{i}+x_{i+1}\right) \quad(0 \leqslant i \leqslant n) .
$$

Define $\hat{z}$ on $\bar{I}$ by

(4.14) $\hat{z}(x):= \begin{cases}\left(\frac{1}{2} h_{0}\right)^{r}, & x_{0} \leqslant x \leqslant p_{0}, \\ \left(\frac{1}{2} h_{i}\right)^{r} \chi\left(\frac{x-p_{i}}{\frac{1}{2} h_{i}}\right), & p_{i} \leqslant x \leqslant x_{i+1}, \quad 0 \leqslant i \leqslant n-1, \\ \left(\frac{1}{2} h_{i+1}\right)^{r} \times\left(\frac{p_{i+1}-x}{\frac{1}{2} h_{i+1}}\right), & x_{i+1} \leqslant x \leqslant p_{i+1}, \quad 0 \leqslant i \leqslant n-1, \\ \left(\frac{1}{2} h_{n}\right)^{r}, & p_{n} \leqslant x \leqslant x_{n+1},\end{cases}$

where

$$
\chi(y):=1-\frac{(2 r-1) !}{[(r-1) !]^{2}} \int_{0}^{y}[z(1-z)]^{r-1} d z .
$$

One may verify that

$$
\begin{cases}\chi(0)=1, \quad \chi(1)=0, & \\ \chi^{(j)}(0)=\chi^{(j)}(1)=0 & (1 \leqslant j \leqslant r-1), \\ \chi^{\prime}(y)<0 & \text { for } 0<y<1, \\ \chi(y)>0 & \text { for } 0 \leqslant y<1,\end{cases}
$$

and so $\chi \in C^{r-1}([0,1])$ and

$$
\mu:=\int_{0}^{1} \chi(y) d y>0 .
$$

Since $\hat{z}$ is piecewise polynomial and globally $C^{r-1}, \hat{z} \in H^{r}(I)$. A straightforward computation yields

$$
\|\hat{z}\|_{r} \leqslant \alpha:=\left\{\left[\sum_{j=0}^{r}\left(\frac{|I|}{2}\right)^{2 r-2 j} \lambda_{j}\right]|I|\right\}^{1 / 2},
$$

where

$$
\lambda_{j}:=\int_{0}^{1} \chi^{(j)}(y) d y .
$$


Note that $\alpha$ is a fixed positive constant, independent of $n$ and the choice of $x_{1}, \ldots, x_{n} \in \bar{I}$. Now define

$$
z(x):=\hat{z}(x) / \alpha
$$

Since $\hat{z}$ vanishes at $x_{1}, \ldots, x_{n}$ and (4.18) holds, we have $z \in$ ker $\mathscr{T}_{n} \cap \mathscr{F}_{0}$.

Another computation yields

$$
\begin{aligned}
\int_{I} z & =\frac{1}{\alpha}(1+\mu)\left(\frac{1}{2} h_{0}\right)^{r+1}+\frac{2 \mu}{\alpha} \sum_{i=1}^{n}\left(\frac{1}{2} h_{i}\right)^{r+1}+\frac{1}{\alpha}(1+\mu)\left(\frac{1}{2} h_{n+1}\right)^{r+1} \\
& \geqslant \lambda \sum_{i=0}^{n+1} h_{i}^{r+1}
\end{aligned}
$$

where

$$
\lambda:=\frac{1}{\alpha 2^{r+1}} \min (1+\mu, 2 \mu)
$$

Since

$$
\sum_{i=0}^{n+1} h_{i}=|I|,
$$

one may use elementary calculus to show that

$$
\sum_{i=0}^{n+1} h_{i}^{r+1} \geqslant|I|\left(\frac{|I|}{n+2}\right)^{r}
$$

Using (4.11), (4.21), and (4.24), there is an $\alpha>0$, independent of $n$ and $x_{1}, \ldots, x_{n}$, such that

$$
r\left(\mathscr{\vartheta}_{n}, S, \mathscr{F}_{0}\right) \geqslant \alpha n^{-r},
$$

proving (i). The rest of the theorem follows from (i) and (4.7) with $N=1$.

Remark 4.1. In Section 6.4 of Part A, it is shown that the optimal error for using $n$ evaluations in numerical quadrature is $\Theta\left(n^{-r}\right)$, provided $\mathscr{F}_{0}=\left\{f \in H^{r}(I)\right.$ : $\left.\left\|f^{(r)}\right\|_{L_{2}(I)} \leqslant 1\right\}$. We are not able to make direct use of this fact, because we must use $\mathscr{F}_{0}=\left\{f \in H^{r}(I):\|f\|_{r} \leqslant 1\right\}$ in order to take advantage of the shift theorem (2.5). Similarly, the results of Sobolev [8] may be used to show that in $N$ dimensions, the optimal error for using $n$ evaluations in numerical quadrature is $\Theta\left(n^{-r / N}\right)$, provided $\mathscr{F}_{0}$ consists of all $f \in H^{r}(\Omega)$ whose $H^{r}(\Omega)$ seminorm is bounded by a fixed constant. In order to use the shift theorem (2.5), we must assume that the $H^{r}(\Omega)$ norm is bounded by a fixed constant. Of course, the two hypotheses are not interchangeable, since any element of $\mathscr{P}_{r-1}$ has $H^{r}(\Omega)$ seminorm of zero; thus we cannot immediately use the results of Sobolev [8] to yield a lower bound of $\Omega\left(n^{-r / N}\right)$ for quadrature (and thus, for the problem $S$ ) in $N$ dimensions for general $N$.

Remark 4.2. Suppose $\mathscr{F}_{0}$ is the unit ball of $C(\bar{\Omega})$ under the sup norm. Since the information $\Re_{n} f$ is well defined for any $f \in C(\bar{\Omega})$, we wish to consider the optimal error behavior of algorithms $\varphi$ using $\Re_{n}$ in this case. Since $\mathscr{F}_{0}$ is not the unit ball of a Hilbert space, (3.21) is not guaranteed to hold. However, it is known that

$$
\inf _{\varphi} e(\varphi)=c \sup _{f \in \operatorname{ker} \vartheta_{n} \cap \mathscr{T}_{0}}\|S f\|_{1}
$$


where $c \in[1,2]$; see Sections 2.2 and 2.3 of Part A. It is possible to show that

$$
\sup _{f \in \operatorname{ker} \Re_{n} \cap \mathscr{F}_{0}}\|S f\|_{1}=\sup _{f \in \mathscr{F}_{0}}\|S f\|_{1}=: \kappa .
$$

Since $S \neq 0, \kappa$ is a positive constant, independent of $n$. Thus for any algorithm $\varphi_{n}$ using $\Re_{n}$, we see that

$$
\kappa \leqslant e\left(\varphi_{n}\right) \leqslant 2 \kappa,
$$

no matter how large $n$ is. So, the minimal condition for $\Re_{n}$ to be defined is not strong enough to yield an algorithm of error less than $\kappa$.

Remark 4.3. Once again, we could consider adaptive standard information, in which $x_{i}$ depends on $x_{j}$ and $f\left(x_{j}\right)$ for $1 \leqslant j \leqslant i-1$. However, for any adaptive standard information $\Re$, one can find nonadaptive standard information $\Re^{\text {non }}$ of the same cardinality for which $r\left(\Re^{\text {non }}, S, \mathscr{F}_{0}\right) \leqslant r\left(\mathscr{T}, S, \mathscr{F}_{0}\right)$; see Section 2.7 of Part A. So, adaptive information does not help.

From Theorems 3.1 and 4.1, we easily conclude

COROLlaRY 4.1. For $N=1$,

$$
\frac{r\left(\mathcal{T}_{n}^{* *}, S, \mathscr{F}_{0}\right)}{r\left(\mathcal{K}_{n}^{*}, S, \mathscr{F}_{0}\right)}=\Theta(n) .
$$

This shows how much we lose by using the standard information $\Re_{n}^{* *}$ instead of the asymptotically optimal information $\Re_{n}^{*}$. The penalty goes to infinity with $n$.

We now consider the computational complexity of using the finite element method with quadrature $\varphi_{n}^{* *}$ to find an $\varepsilon$-approximation to $S$, in the case $N=1$. By (iii) of Theorem 4.1, to guarantee $e\left(\varphi_{n}^{* *}\right) \leqslant \varepsilon$, we require

$$
n=\Theta\left(\varepsilon^{-1 / r}\right) \text { as } \varepsilon \rightarrow 0 .
$$

Again, $\varphi_{n}^{* *}$ is linear, i.e., there exist $g_{i} \in H_{0}^{1}(\Omega)$ such that

$$
\varphi_{n}^{* *}\left(\mathcal{T}_{n}^{* *} f\right)=\sum_{i=1}^{n} f\left(x_{i}\right) g_{i} .
$$

Let us agree to compute the $g_{i}$ in advance and not count the cost of this precomputation. Assume that the evaluation of $f$ has finite cost. We then find that the complexity of using the finite element method with quadrature to compute an $\varepsilon$-approximation is

$$
\operatorname{COMP}\left(\varphi_{n}^{* *}, \varepsilon\right)=\Theta\left(\varepsilon^{-1 / r}\right) \quad \text { as } \varepsilon \rightarrow 0 .
$$

Since $\varphi_{n}^{* *}$ is a linear asymptotically optimal error algorithm using $\Re_{n}$, we see that

$$
\operatorname{COMP}^{* *}(S, \varepsilon)=\inf _{\varphi} \operatorname{COMP}(\varphi, \varepsilon)=\Theta\left(\varepsilon^{-1 / r}\right) \quad \text { as } \varepsilon \rightarrow 0
$$

the infimum being taken over all $\varphi$ using standard information and for which $e(\varphi) \leqslant \varepsilon$. Thus $\varphi_{n}^{* *}$ is of asymptotically optimal complexity using standard information

Comparing (3.47) and (4.32), we find

COROLlaRY 4.2. If $N=1$,

$$
\frac{\operatorname{COMP}^{* *}(S, \varepsilon)}{\operatorname{COMP}(S, \varepsilon)}=\Theta\left(\left(\frac{1}{\varepsilon}\right)^{1 /\left(r^{2}+r\right)}\right) \quad \text { as } \varepsilon \rightarrow 0 .
$$


This is another measure of the penalty for using standard information.

As in Section 3, we may wish to avoid the assumption of preconditioning. In this case, we once again have to solve a linear system involving a large sparse matrix. However, for the case $N=1$, which we are considering, the linear system may once again be solved in linear time, so that the finite element method with quadrature remains an asymptotically optimal complexity algorithm.

Acknowledgements. I would like to thank Dr. A. B. Stephens (U. S. Naval Surface Weapons Center, White Oak, Maryland), Professor J. F. Traub (Columbia University), and Professor H. Woźniakowski (University of Warsaw) for their comments, suggestions, and encouragement. In addition, I would like to thank the referee for his suggestions, which improved the exposition of this paper.

Department of Mathematics

University of Maryland Baltimore County

Catonsville, Maryland 21228

1. S. Agmon, Lectures on Elliptic Boundary Value Problems, Van Nostrand, Princeton, N. J., 1965.

2. I. BABUSKA \& A. K. AzIz, "Survey lectures on the mathematical foundations of the finite element method," in The Mathematical Foundations of the Finite Element Method with Applications to Partial Differential Equations (A. K. Aziz, ed.), Academic Press, New York, 1972.

3. P. G. Ciarlet, The Finite Element Method for Elliptic Problems, North-Holland, Amsterdam, 1978.

4. P. G. Ciarlet \& P. A. Raviart, "Interpolation theory over curved elements," Comput. Methods Appl. Mech. Engrg., v. 1, 1972, pp. 217-249.

5. J. W. Jerome, "Asymptotic estimates of the $L_{2}$-width," J. Math. Anal. Appl., v. 22, 1968, pp. 449-464.

6. J. W. Jerome, “On $n$-widths in Sobolev spaces and elliptic boundary value problems," J. Math. Anal. Appl., v. 29, 1970, pp. 201-215.

7. M. H. SchulTz, "Multivariate spline functions and elliptic problems," in Approximation with Special Emphasis on Spline Functions (J. J. Schoenberg, ed.), Academic Press, New York, 1969.

8. S. L. Sobolev, "On the order of convergence of cubature formulas," Dokl. Akad. Nauk SSSR, v. 162, 1965, pp. 1005-1008; English transl. in Soviet Math. Dokl., v. 6, 1965, pp. 808-812.

9. J. F. Traub \& H. Woźniakowski, A General Theory of Optimal Algorithms, Academic Press, New York, 1980. 